\title{
CORRECTION
}

View Article Online

View Journal | View Issue

Check for updates

Cite this: Mater. Chem. Front. $2022,6,807$

DOI: $10.1039 / \mathrm{d} 2 \mathrm{qm} 90016 \mathrm{e}$

rsc.li/frontiers-materials

\section{Correction: Recent advances in material design and reactor engineering for electrocatalytic ambient nitrogen fixation}

\author{
Tariq Ali, ${ }^{\text {ab }}$ Niaz Muhammad, ${ }^{c}$ Yijun Qian, ${ }^{d}$ Sisi Liu, ${ }^{a}$ Sai Wang, ${ }^{\text {a }}$ Mengfan Wang ${ }^{\text {*a }}$ \\ Tao Qian ${ }^{\mathrm{e}}$ and Chenglin Yan ${ }^{\mathrm{a}}$
}

Correction for 'Recent advances in material design and reactor engineering for electrocatalytic ambient nitrogen fixation' by Tariq Ali et al., Mater. Chem. Front., 2022, DOI: 10.1039/d1qm01620b.

The authors regret that one of the author names (Yijun Qian) was incorrectly spelled in the original manuscript. The corrected list of authors and affiliations for this paper is as shown above.

The Royal Society of Chemistry apologises for these errors and any consequent inconvenience to authors and readers.

\footnotetext{
${ }^{a}$ College of Energy, Key Laboratory of Advanced Carbon Materials and Wearable Energy Technologies of Jiangsu Province, Soochow University, Suzhou 215006, China. E-mail:wangsai@suda.edu.cn, mfwang1204@suda.edu.cn

${ }^{b}$ Key Laboratory of the Ministry of Education for Advanced Catalysis Materials, Department of Chemistry, Zhejiang Normal University, Jinhua 321004, China

${ }^{c}$ Department of Chemistry, Abdul Wali Khan University, Mardan, Pakistan

${ }^{d}$ Deakin University, Institute for Frontier Materials, Waurn Ponds Campus, Locked Bag 20000, Geelong, Victoria 3220, Australia

${ }^{e}$ School of Chemistry and Chemical Engineering, Nantong University, Nantong 226019, China
} 